\title{
Photonic millimeter-wave frequency multiplication based on cascaded four-wave mixing and polarization pulling
}

\author{
B. Vidal \\ Nanophotonics Technology Center, Universitat Politècnica de València, Valencia 46022, Spain \\ (bvidal@dcom.upv.es)
}

Received September 5, 2012; revised October 24, 2012; accepted October 31, 2012; posted November 1, 2012 (Doc. ID 175676); published December 5, 2012

\begin{abstract}
A technique for the frequency multiplication of microwave signals based on the combination of two optical nonlinear phenomena in a single nonlinear fiber is investigated. Multiple four-wave mixing is used to generate harmonics on an externally modulated optical carrier while polarization pulling through stimulated Brillouin scattering is used to filter the desired harmonics. Microwave signals in the $60 \mathrm{GHz}$ region are generated showing harmonic frequency multiplication factors of up to 25 with a suppression of undesired harmonics better than $20 \mathrm{~dB}$. (C) 2012 Optical Society of America

OCIS codes: $\quad 060.0060,190.0190,190.4380,070.1170$.
\end{abstract}

Frequency multipliers are key components in instrumentation and communication applications. As these systems move toward higher frequencies to avoid spectrum congestion and to allow for the provision of high bit rate services, the implementation of broadband frequency mixers is getting more difficult. Photonic technology offers an alternative way to develop microwave and millimeterwave mixers with wide bandwidth, potential to be integrated with radio-over-fiber distribution systems, and immunity to electromagnetic interference. Different optical approaches have been proposed using reciprocating modulation [1] or different configurations of cascaded external modulators [2]. However, the mixing multiplication factors obtained with these techniques remain low in comparison with the current performance of purely electronic mixers. A different approach is based on optical nonlinear effects, which can be employed to enhance microwave harmonics and upconvert signals. Stimulated Brillouin Scattering (SBS) was used to generate millimeter-wave signals in the W-band from a $7.5 \mathrm{GHz}$ tone (i.e., a multiplication factor of 14) [3] and four-wave mixing (FWM) has also been proposed both in fiber [4] and semiconductor optical amplifiers [5] to implement microwave mixers with multiplication factors up to 6 and 3, respectively. A more complex system [] $]$ based on the joint operation of polarization modulation, FWM, and SBS assisted filtering allowed a higher multiplication factor (12).

FWM is a third-order parametric process which involves the interaction of four different copropagating signals (two strong pump waves, one weak signal, and a weak idler in the nondegenerated FWM case) [7]. If the performance of this process is sufficiently optimized, it can result in new pumps being generated and new FWM processes in what is known as multiple or cascaded FWM $[\underline{8}, \underline{9}]$.

In this Letter, cascaded FWM in a highly nonlinear fiber (HNLF) is used to generate new harmonics of the microwave signal to be upconverted. Additionally, the nonlinear medium is reused to actively filter a pair of sidebands with narrow selectivity through polarization pulling by SBS and a polarizer.
The technique is based on the amplitude modulation of a CW optical carrier (Laser $1,1561 \mathrm{~nm}$ ) used as pump wave by the microwave tone to be frequency multiplied using an external electro-optic Mach-Zehnder modulator, as shown in Fig. 1. The optical signal is boosted by the combination of a preamplifier and a high-power EDFA to reach an optical power of $0.5 \mathrm{~W}$. After an isolator, the signal goes through a reel of 900 meters of $\operatorname{HNLF}\left(\gamma=10.8 \mathrm{~W}^{-1} \mathrm{~km}^{-1}, \lambda_{0}=1562 \mathrm{~nm}, S_{D}=0.018 \mathrm{ps} /\right.$ $\left.\left(\mathrm{nm}^{2} \cdot \mathrm{km}\right)\right)$.

In the HNLF, cascaded FWM amplifies the harmonics generated by the modulator and generates new sidebands around the pump carrier $[\underline{8}, \underline{9}]$. Figure $\underline{2}$ shows the spectrum of an optical signal with and without cascaded FWM for a microwave input signal of $5.5 \mathrm{GHz}$. The solid line shows the optical spectrum at the output of the HNLF when a low power signal $(0.01 \mathrm{~W})$ impinges into the fiber, avoiding the generation of nonlinear effects. The dashed line shows the spectra for an optical power of $0.5 \mathrm{~W}$.

Once cascaded FWM has generated new optical harmonics from the sidebands provided by the amplitude modulation, a pair of sidebands have to be selected. Instead of the use of fixed filters, the nonlinear medium

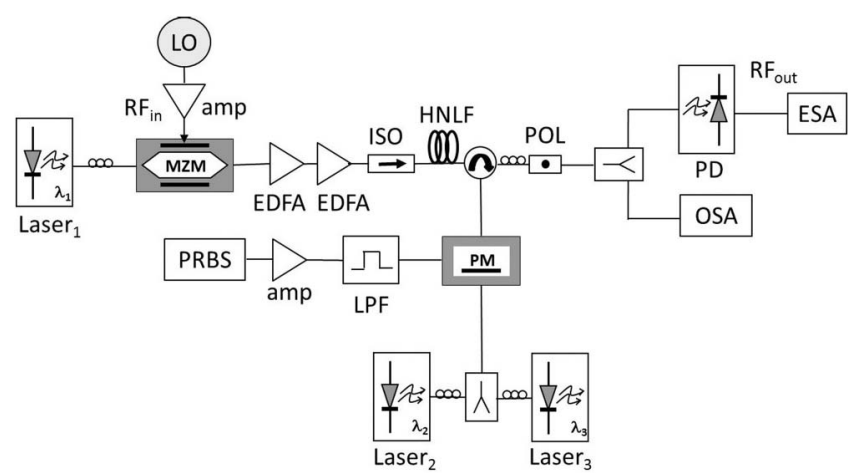

Fig. 1. Experimental setup for the photonic microwave harmonic mixer based on FWM and SBS polarization pulling. ISO, optical isolator; LPF, microwave lowpass filter; POL, optical polarizer; ESA, electrical spectrum analyzer; OSA, optical spectrum analyzer. 


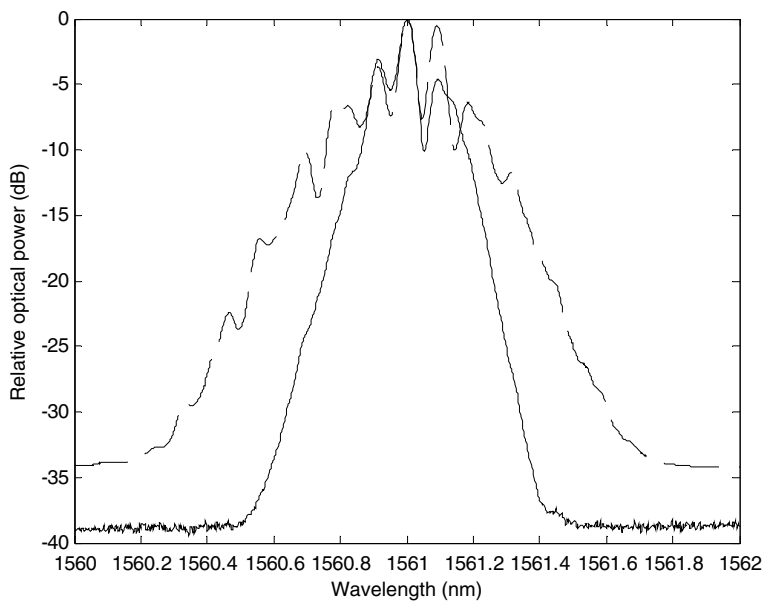

Fig. 2. Optical spectra at the output of the HNLF for $0.01 \mathrm{~W}$ (solid) and $0.5 \mathrm{~W}$ (dashed).

allows active filtering through SBS polarization pulling. This eases the tuning of the frequency multiplied microwave harmonic.

In SBS two counterpropagating waves interfere and, through electrostriction, an acoustic wave generates a traveling grating that couples both optical signals, resulting in a transfer of energy from the wave of higher frequency to the lower one. Thus, SBS is strongly polarization dependent due to the need of interference between both waves. If both the pump and the signal do not have their states of polarization (SOP) properly aligned, SBS amplification, although not getting maximum gain, results in the pull of the signal SOP toward the pump SOP [10]. The narrowband nature of the Brillouin gain bandwidth allows polarization control with extreme selectivity in wavelength [11].

The SOP of two sidebands generated from cascaded FWM can be modified through SBS polarization pulling. If these two sidebands are cross-polarized in relation to the amplitude modulated waves, an optical polarizer can be used to attenuate all the signals but the two sidebands of interest. Additionally, SBS amplification will result in a certain gain for the selected sidebands.

In the setup of Fig. 1 two CW optical sources $\left(\right.$ Laser $_{2}$ and Laser $_{3}, 1560.65$ and $1561.18 \mathrm{~nm}$, respectively) are used as pump waves for polarization pulling. These pumps are phase modulated to control the bandwidth of the Brillouin gain. In particular, the pump waves are broadened using a phase modulator (PM) driven by a lowpass signal. This lowpass signal is generated by the filtering of a $2^{7}-1 \mathrm{NRZ}$ pseudorandom binary sequence at 11 Gbps (PRBS) with a $500 \mathrm{MHz}$ low-pass filter. The optical power at the input of the circulator is around $14 \mathrm{dBm}$.

The modulated pumps counterpropagate the FWM products through an optical circulator. A fiber in-line optical polarizer (with an extinction ratio of $34 \mathrm{~dB}$ ) is used to filter the desired sidebands. Finally, the optical signal is split by a $10 \%-90 \%$ optical coupler. The $10 \%$ output is used to monitor the optical spectrum (using an optical spectrum analyzer, OSA) while the other one feeds a $75 \mathrm{GHz}$ photodetector connected without microwave amplifiers to an electrical spectrum analyzer (ESA, R\&S FSQ40) with an external V-band mixer (R\&S FS-Z75) to evaluate the generated microwave signals.

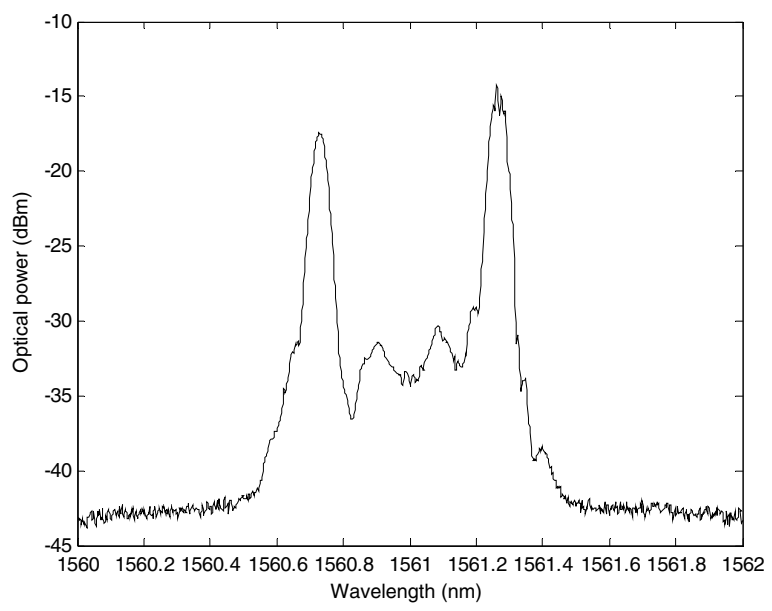

Fig. 3. Optical spectrum measured after the optical polarizer, showing narrowband filtering of the \pm 6 th sidebands through polarization pulling and a polarizer.

When the frequency of the pump waves is adjusted to be the Brillouin frequency shift $\left(v_{B} \approx 9.6 \mathrm{GHz}\right.$ in HNLF) higher than the two selected sidebands and after proper adjusting of the polarization states of pumps and signals, polarization rotation of the desired sidebands is achieved. Figure 3 shows the optical signal after the polarizer. Sideband filtering through polarization pulling for an input microwave tone of $5.559 \mathrm{GHz}$ can be seen. Undesired sidebands are attenuated more than $13 \mathrm{~dB}$ in relation to the selected harmonics.

The microwave spectrum obtained from the signal of Fig. 3 can be seen in Fig. 4. A microwave tone of frequency $66.71 \mathrm{GHz}$ (corresponding to a multiplication factor of 12) is generated with a suppression of undesired harmonics higher than $24 \mathrm{~dB}$. The phase noise at an offset of $10 \mathrm{KHz}$ has been measured with the spectrum analyser (R\&S FSQ40) and it is $-73.2 \mathrm{dBc} / \mathrm{Hz}$. The phase noise of the microwave tone at the input of the system was $-95.6 \mathrm{dBc} / \mathrm{Hz}$ at $10 \mathrm{KHz}$ offset. This relation follows the theoretical prediction of a phase noise increase according to $20 \log N(\mathrm{~dB})$ for the $N$ th harmonic (i.e., a measured phase noise degradation of $22.4 \mathrm{~dB}$ versus

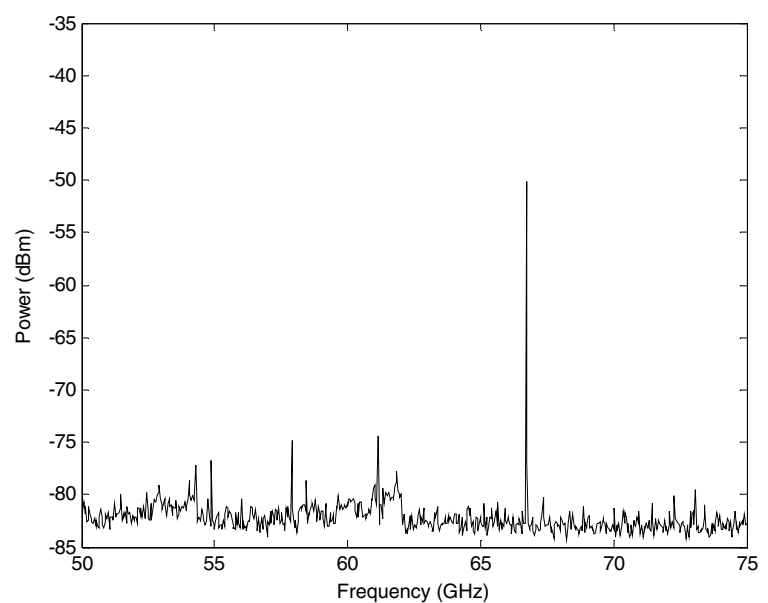

Fig. 4. Microwave spectrum generated from the heterodyning of two cascaded FWM-generated sidebands filtered through polarization pulling with an input microwave signal of $5.5 \mathrm{GHz}$ and a multiplication factor of 12 . 


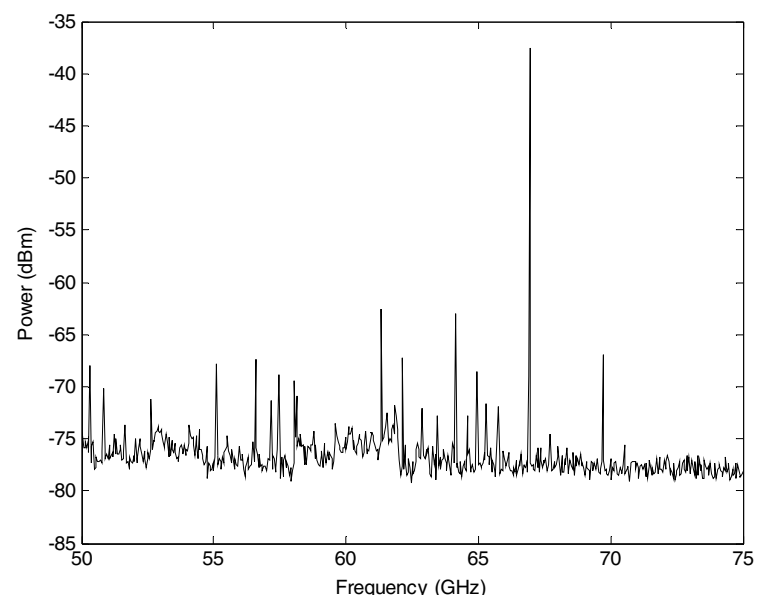

(a)

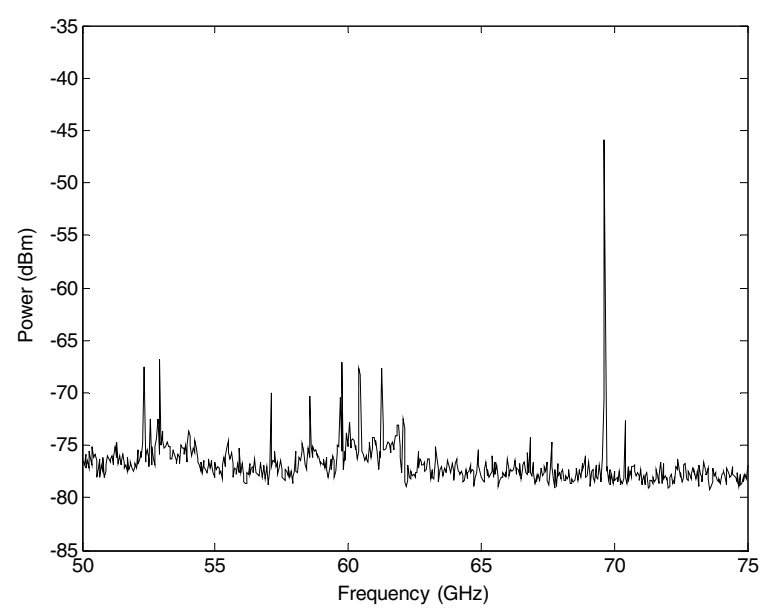

(b)

Fig. 5. Measured microwave spectrum for an input microwave signal of $2.7 \mathrm{GHz}$ and a multiplication factor of (a) 24 and (b) 25 .

a theoretical prediction of $21.6 \mathrm{~dB}$ ). Therefore, the performance in terms of phase noise is similar to microwave harmonic mixers.

Fig. 5 shows the capability of the technique to be tuned both in terms of frequency and multiplication factor thanks to active filtering. Figs. $5 \mathrm{a}$ and $5 \mathrm{~b}$ show the microwave spectrum for multiplication factors of 24 $(66.95 \mathrm{GHz})$ and $25(69.63 \mathrm{GHz})$, respectively, and for an input signal of $2.78 \mathrm{GHz}$. The suppression of undesired harmonics is 25.3 for the former and $21.1 \mathrm{~dB}$ for the latter. The difference in the harmonic suppression is due to errors in the adjusting of the polarization filtering.

In conclusion, a new technique for the photonic frequency multiplication of microwave signals has been proposed and demonstrated. The combination of two nonlinear effects (cascaded FWM and SBS) in a single HNLF offers a flexible and efficient method for the generation of millimeter-wave signals. The frequency and/or the harmonic number can be dynamically selected with the proposed setup. The tuning range is only limited by the photodiode since the technique relies only upon optical nonlinear effects. Actually, enhanced cascaded FWM processes are expected from input signals of higher frequency [12].

This research was supported in part by the Spanish Ministerio de Economía y Competitividad through TEC2009-08078 and TEC2012-35797 projects.

\section{References}

1. T. Kawanishi, S. Oikawa, K. Yoshiara, T. Sakamoto, S. Shinada, and M. Izutsu, IEEE Photonics Technol. Lett. 17, 669 (2005).

2. W. Li and J. P. Yao, IEEE Trans. Microwave Theory Tech. 58, 3259 (2010).

3. B. Vidal, P. G. Huggard, B. N. Ellison, and N. J. Gomes, IEEE Electron. Lett. 46, 1449 (2010).

4. A. Wiberg, P. Pérez-Millán, M. V. Andrés, and P. O. Hedekvist, J. Lightwave Technol. 24, 329 (2006).

5. Q. Wang, H. Rideout, F. Zeng, and J. Yao, IEEE Photonics Technol. Lett. 18, 2460 (2006).

6. W. Li and J. P. Yao, IEEE Photon. J. 2, 954 (2010).

7. G. P. Agrawal, Nonlinear Fiber Optics (Academic, 2007).

8. C. J. McKinstrie and M. G. Raymer, Opt. Express 14, 9600 (2006).

9. A. Cerqueira, J. M. Chavez Boggio, A. A. Rieznik, H. E. Hernández-Figueroa, H. L. Fragnito, and J. C. Knight, Opt. Express 16, 2816 (2008).

10. L. Thévenaz, A. Zadok, A. Eyal, and M. Tur, in Optical Fiber Communications, OSA Technical Digest CD (Optical Society of America, 2008) paper OML7.

11. A. Wide, M. Tur, and A. Zadok, Opt. Express 19, 21945 (2011).

12. J. Hansryd, P. A. Andrekson, M. Westlund, J. Li, and P. O. Hedekvist, IEEE J. Sel. Top. Quantum Electron. 8, 506 (2002). 